\title{
Implementation of wellness into a cardiothoracic training program: A checklist for a wellness policy
}

\author{
Romulo Fajardo, MD, ${ }^{\mathrm{a}}$ Ara Vaporciyan, MD, ${ }^{\mathrm{b}}$ Sandra Starnes, $\mathrm{MD},{ }^{\mathrm{c}}$ and Cherie P. Erkmen, $\mathrm{MD}^{\mathrm{d}}$
}

Physician wellness impacts patient care and education of trainees. Lack of physician wellness and burnout is characterized by emotional exhaustion, depersonalization, and a low sense of accomplishment. ${ }^{1}$ Burnout puts physicians at risk for substance abuse, intent to leave medical practice, and suicide. ${ }^{2}$ Unfortunately, $50 \%$ to $70 \%$ of physician trainees report burnout, ${ }^{3} 29 \%$ experience depression, ${ }^{4}$ and $6 \%$ to $12 \%$ report suicidal ideation. ${ }^{1}$ The physical demands and long work hours of surgical practice have been attributed to greater rates of burnout among surgeons. ${ }^{5}$ Cardiothoracic surgeons have the greatest number of hours in the operating room, greater positive depression screening assessment, and lower mental quality of life score in comparison with other surgical specialties. ${ }^{5,6}$ In addition to individual dangers, burnout can be linked to medical errors, problems with professionalism, and adverse patient outcomes. $^{7-9}$ Attributable cost of physician burnout in the United States is approximately $\$ 4.6$ billion dollars annually, which equates to an annual cost of $\$ 7600$ per physician. ${ }^{9}$

These alarming statistics have prompted the Accreditation Council for Graduate Medical Education (ACGME) to take meaningful action. In 2015, the ACGME organized the first Symposium on Physician Well-Being to promote change in physician wellness. ${ }^{10}$ In 2016, the Association of American Medical Colleges and National Academy of Medicine formed the Action Collaborative on Clinician Well-Being and Resilience. In 2017, the ACGME revised its common program requirements to include specific action for all residency programs (Figure 1). Many training programs have implemented these common program requirements. However, there are no guidelines for implementation into cardiothoracic training programs. The purpose of this work is to help organize an approach to wellness for cardiothoracic surgery training programs. We have

\footnotetext{
From the ${ }^{\mathrm{a}}$ Department of General Surgery, Temple University Hospital, Philadelphia, $\mathrm{Pa} ;{ }^{\mathrm{b}}$ Department of Thoracic and Cardiovascular Surgery, University of Texas MD Anderson Cancer Center, Houston, Tex; ${ }^{\mathrm{c}}$ Division of Thoracic Surgery, University of Cincinnati College of Medicine, Cincinnati, Ohio; and ${ }^{\mathrm{d}}$ Department of Thoracic Medicine and Surgery, Lewis Katz School of Medicine at Temple University, Philadelphia, $\mathrm{Pa}$.

Received for publication Oct 24, 2019; revisions received April 21, 2020; accepted for publication April 23, 2020; available ahead of print June 27, 2020.

Address for reprints: Romulo Fajardo, MD, Department of General Surgery, Temple University Hospital, 3401 N Broad St, Parkinson Pavilion, Suite 400, Philadelphia, PA 19140 (E-mail: romuloandre.fajardo@tuhs.temple.edu).

J Thorac Cardiovasc Surg 2021;161:1979-86

$0022-5223 / \$ 36.00$

Copyright (c) 2020 by The American Association for Thoracic Surgery

https://doi.org/10.1016/j.jtcvs.2020.04.186
}

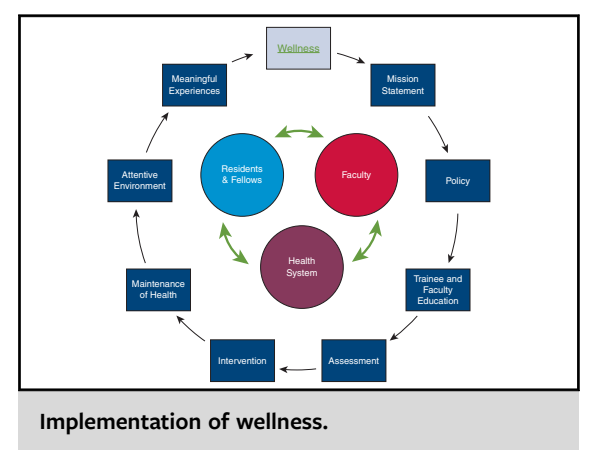

\begin{abstract}
CENTRAL MESSAGE
We have developed a wellness

policy checklist and sample well-

ness policy to help guide

cardiothoracic surgery training

programs with wellness imple-

mentation that adheres to the

ACGME requirements.
\end{abstract}

See Commentaries on pages 1987 and 1988.

developed a checklist to assess the culture of wellness within a training program and a sample wellness policy that encompasses ACGME Well-Being common program requirements and practices from other specialties.

\section{METHODS}

An online web search using the key words wellness, physician well-being, burnout, and fatigue was conducted and completed by September 25, 2019. Literature and established guidelines for wellness were reviewed from resources of the ACGME, Association of American Medical Colleges, American Medical Association (AMA), National Academy of Medicine, American College of Surgeons (ACS), American College of Obstetricians and Gynecologists, Johns Hopkins Medicine, Mayo Clinic, Oregon Health \& Science University, and the University of Colorado websites. Recommendations were developed for programs wishing to implement wellness and adherence to ACGME requirements.

\section{RESULTS}

A wellness program for cardiothoracic training programs must adhere to common program requirements of the ACGME (Figure 1). Each ACGME training program has to "enhance the meaning that each resident finds in the experience of being a physician." Wellness includes being challenged, building competence, autonomy, achieving 
VI.C. Well-Being

VI.C.1.a) This responsibility must include: efforts to enhance the meaning that each resident finds in the experience of being a physician, including protecting time with patients, minimizing non-physician obligations, providing administrative support, promoting progressive autonomy and flexibility, and enhancing professional relationships; (Core)

VI.C.1.b) This responsibility must include: attention to scheduling, work intensity, and work compression that impacts resident well-being; (Core)

VI.C.1.C) This responsibility must include: evaluating workplace safety data and addressing the safety of residents and faculty members; (Core) Examples of data sources include (but are not limited to) Joint Commission and Occupational Safety and Health Administration (OSHA) reports.

VI.C.1.d) This responsibility must include: policies and programs that encourage optimal resident and faculty member well-being; and, (Core)

VI.C.1.d.(1) Residents must be given the opportunity to attend medical, mental health, and dental care appointments, including those scheduled during their working hours. (Core)

VI.C.1.e) This responsibility must include: attention to resident and faculty member burnout, depression, and substance abuse. The program, in partnership with its Sponsoring Institution, must educate faculty members and residents in identification of the symptoms of burnout, depression, and substance abuse, including means to assist those who experience these conditions. Residents and faculty members must also be educated to recognize those symptoms in themselves and how to seek appropriate care.

VI.C.1.e).(1) The program, in partnership with its Sponsoring Institution, must: encourage residents and faculty members to alert the program director or other designated personnel or programs when they are concerned that another resident, fellow, or faculty member may be displaying signs of burnout, depression, substance abuse, suicidal ideation, or potential for violence; (Core)

VI.C.1.e).(2) The program, in partnership with its Sponsoring Institution, must: provide access to appropriate tools for self-screening; and, (Core)

X VI.C.1.e).(3) The program, in partnership with its Sponsoring Institution, must: provide access to confidential, affordable mental health assessment, counseling, and treatment, including access to urgent and emergent care 24 hours a day, seven days a week. (Core)

VI.C.2. There are circumstances in which residents may be unable to attend work, including but not limited to fatigue, illness, and family emergencies. Each program must have policies and procedures in place that ensure coverage of patient care in the event that a resident may be unable to perform their patient care responsibilities. These policies must be implemented without fear of negative consequences for the resident who is unable to provide the clinical work.

(Acgme.org, 2020)

Available at: https://www.acgme.org/Portals/0/PFAssets/ProgramRequirements/CPRResidency2019.pdf

[Accessed 3 Mar. 2020]

FIGURE 1. Accreditation Council for Graduate Medical Education Well-Being Requirements 2019.

meaningful personal and professional success, and strong social relatedness to work. ${ }^{3,11}$ Training programs must pay attention to scheduling (section VI.C.1.b), which includes duty-hour adherence, but also consideration of workload. ${ }^{12}$ Workload can be measured by patient census, ${ }^{13}$ patient complexity,${ }^{14}$ or paging data. ${ }^{15}$ Work-related stress, activities with marginal educational value (phlebotomy or transportation), and the burden of medical documentation can increase the perception of workload. ${ }^{12}$ Currently, there is no consensus on measurement of meaningful work or resident workload. Nevertheless, cardiothoracic training programs are responsible for providing a schedule that enhances the meaning that each resident finds in being a physician.

ACGME Well-Being Requirements also include adherence to workplace and personal safety standards (VI.C.1.c). Safety efforts have been focused on fatiguerelated harm like motor vehicle accidents and needle sticks. ${ }^{16}$ Training programs and hospitals should provide transportation to trainees who feel too fatigued to safely 
navigate home. ${ }^{16}$ The ACGME Well-Being Requirements extend beyond the workplace and mandate sufficient time away from work to manage individual wellness including medical, mental health, and dental appointments (VI.C.1.d). The AMA and ACS suggest training programs to foster a multifaceted approach to individual wellness through nutrition, fitness, emotional health, preventative care, financial health, and mindset/behavior adaptability. ${ }^{11,17}$

ACGME-accredited training programs must include a wellness education program for residents and faculty (VI.C.1.e). Wellness education should include definition of wellness, fatigue, and burnout ${ }^{3,18}$ and harmful sequelae, including substance abuse, unprofessionalism, depression, and suicide. ${ }^{9}$ Faculty and residents should receive education on fatigue (symptoms, recognition in themselves and others, mitigation, and treatment). ${ }^{19-21}$ Faculty and trainees should be able to identify and seek help for burnout, depression, and substance abuse, ${ }^{22}$ in themselves and others. ACGME Well-Being Requirements specifically mandate residents and faculty members alert the program director or other designated personnel about any resident, fellow, or faculty member experiencing burnout, depression, substance abuse, suicidal ideation, or potential for violence. ACGME requirements for wellness education must also include tools for well-being assessment, selfscreening, and access to mental health services 24 hours a day, 7 days a week (VI.C.1.e).

ACGME Well-Being Requirements mandate policies for residents who may be unable to perform patient care responsibilities (VI.C.2.). Programs should prepare for possible short- and long-term absences. Training programs must ensure safe patient care but also protect those covering for an absent resident. Developing flexible and emergency scheduling protocols before an absence may facilitate patient care and decrease resident stress. ${ }^{23}$

\section{DISCUSSION}

The addition of well-being to ACGME core requirements places direct responsibility of resident wellness on training programs. The requirements are challenging to implement in cardiothoracic surgery training programs for several reasons. First, cardiothoracic surgery is a culture that has traditionally placed the responsibility of wellness on individual surgeons, not on training programs. Many individuals within our field have been willing to sacrifice wellness to achieve the goal of becoming cardiothoracic surgeons. Second, the ACGME does not provide recommendations on how to implement wellness. The resources needed for education, policy development, and service reorganization to foster wellness are not addressed. Third, there is no framework in cardiothoracic surgery to implement wellness. To address this last barrier, we propose 2 tools to guide the development of a comprehensive wellness program that meets the new ACGME Well-Being Requirements: (1) Checklist of essential components of a comprehensive wellness program (Figure 2). The checklist will guide compliance with ACGME requirements but also allow for program-specific adaptations based on each program's local culture of wellness, resources, and current status of wellness implementation. (2) Sample wellness policy template (Figure 3,A-D). Programs may customize this template by filling in the blanks that identify appropriate personnel, dates, and resources. The sample policy includes direct links to educational, assessment, and treatment resources. ${ }^{17,24}$ Our hope is that these tools will help cardiothoracic training programs assess and enhance their approach to resident wellness.

One of the first steps in developing a wellness program should be the development of a mission statement that defines wellness as it relates to the cardiothoracic training program. The mission statement should also include specific language about the level of commitment the program has to promote the defined environment of wellness (Figure 3, $A-D)$. The National Academy of Medicine has examples of wellness mission statements from training programs and health organizations. ${ }^{25}$

Cardiothoracic training programs should develop a wellness policy founded on the mission statement (Figure 3, $A-D)$. A wellness policy should identify a governing body that will uphold the policy. The governing body should consist of program director(s), section chief(s), department chairs, Graduate Medical Education officials, and local expertise in wellness including hospital wellness officers, members of wellness committees, and representatives from psychiatry who will give a larger perspective of existing resources for wellness (Figure 4). Most importantly, a resident or fellow representative should participate in the governance of wellness. The stakeholders should have defined roles and responsibilities outlined in a wellness policy. As a whole, the governing body should be responsible for dissemination, facilitation, adherence, and maintenance of the policy.

A wellness policy should include an education curriculum for residents and faculty. A wellness curriculum should define wellness, fatigue, and burnout as required by the ACGME. ${ }^{3,18}$ To engage faculty and residents, training programs should encourage discussion about wellness, namely how trainees define meaningful work, workload, and health/ safety away from work. Programs should address fatigue, the symptoms of fatigue, and specific times when residents and faculty are at risk of fatigue. A wellness curriculum should include the relationship between fatigue and burnout and how they place surgeons at risk of substance abuse, unprofessionalism, depression, and suicide. ${ }^{9}$ The Thoracic Surgery Resident Association and Thoracic Surgery 
Develop a mission statement specific to the program

Develop a wellness policy founded on the mission statement

- Identify key stakeholders

- Establish a governing body or appoint the responsibility to an existing committee

- Define how adherence to the wellness policy will be monitored and enforced

Develop resident and faculty education

- Importance of wellness

- Defining and recognizing symptoms of fatigue, burnout, and substance abuse

- Reporting of concerns to the Program Director

- Determine continuing education, content, and frequency

Provide tools for self- screening and develop a privacy policy on how to utilize and protect the results

Enhance the resident/trainee experience of being a physician

- Meaningful challenges

○ Building competence

- Sense of achievement

- Social relatedness to the work

- Adequate sleep and time away from work

Foster an environment for attention and maintenance of one's health care

- Time and resources to attend medical, mental health, and dental appointments

- Safe work environment

- Access to water and healthy food

- Safe transportation

- Adequate sleep facilities

- Accessible reporting and intervention with burnout

- 24-hour access to mental health resources

- Develop a crisis plan for the training program in the event of a severe illness, absence, or death (coverage, counseling for colleagues)

Develop a crisis plan for the training program in the event of a severe illness, absence, or death (coverage, counseling for colleagues)

FIGURE 2. Checklist for implementation of wellness in a cardiothoracic surgery training program.

Directors Association and the ACS have developed educational content on fatigue. ${ }^{17,24}$ In addition, faculty and trainees must receive education on the following: (1) Strategies for individuals to identify the symptoms of fatigue and burnout in themselves and others. ${ }^{19-21}$ (2) Means to seek help for themselves or a colleague in need including a list of mental health services. (3) Notification of the program director if a trainee or faculty member appears fatigued, burned out, or at risk of harm to patients, themselves, or others. Programs will have to decide if the curriculum should vary between residents and faculty in content, approach, or time. Once a wellness curriculum is 
Cardiac and Thoracic Surgery Sample Wellness Policy

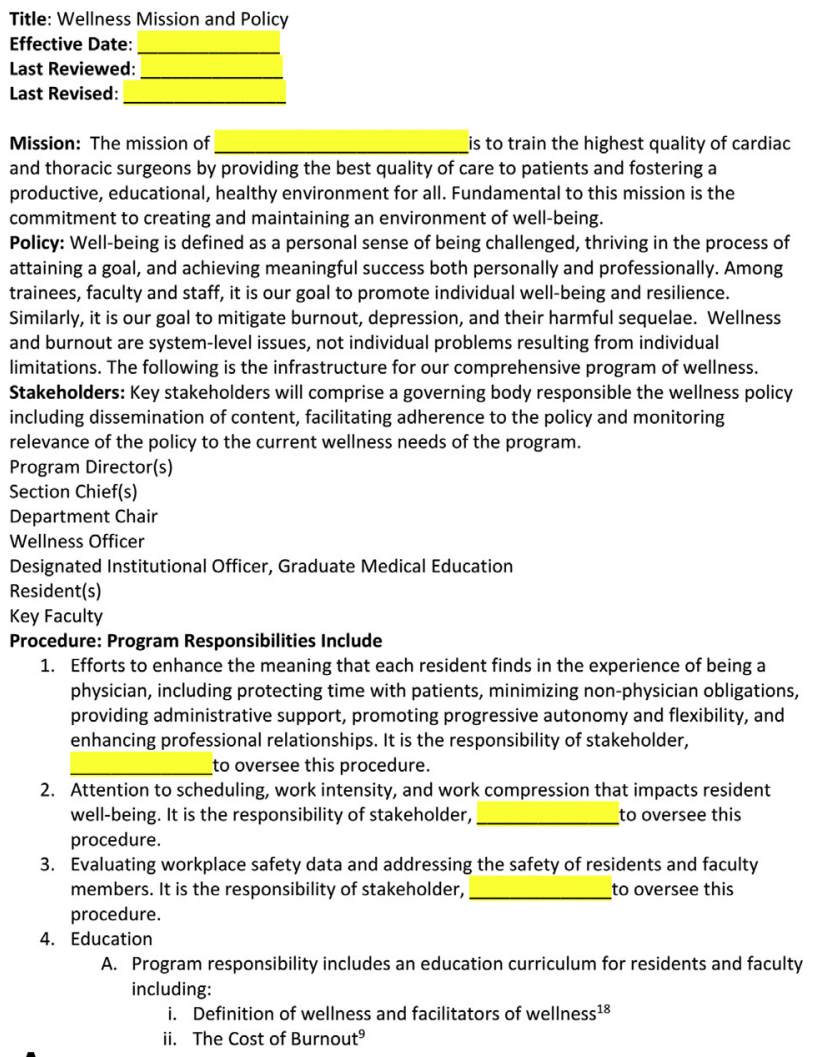

A

FIGURE 3. A-D, Cardiothoracic surgery sample wellness policy. Blank fields to be completed by the cardiothoracic surgery training program. iv. Fatigue: Recognizing Signs of Fatigue and Fatigue Mitigation

1. https://edhub.ama-assn.org/steps-forward teachersguide1.pdf

3. https://www.youtube.com/watch?v=19GRxF9qEBA

4. https://www.facs.org/member-services/surgeon-wellbeing

5. https://www.tsranet.org/wpcontent/uploads/2013/01/Fighting_Fatigue.pdf

v. Steps to seek help for yourself or other

vi. Notification of Program Director of wellness concerns in self or others B. This wellness curriculum should be delivered to residents and faculty annually.

C. It is the responsibility of stakeholder, , to ensure that this content is relevant and delivered on an annual basis.

A. Wellness will be assessed at minimum every 6 months during the semi-annua resident review.

i. Assessment includes questions about feeling challenged, increased competency, increased autonomy, value in their work, sleep and time away from work. ${ }^{18}$

i. Assessment will also include questions about fatigue, burnout, substance abuse in themselves or in their environment.

B. Screening and assessments tools are available to the residents and faculty.

i. Mayo 9 question screening: https://www. mededwebs.com/residentand-fellow-well-being-index

ii. American Medical Association-Mini-Z Burnout Inventory (Screening/Survey Instrument ) https://www.stepsforward.org/Static/images/modules/15/downloadable Mini\%20Z\%20burnout\%20survey.docx

iii. Maslach Burnout 22 question assessment: https://www.mindgarden.com/315-mbi-human-servicessurvey-medical-personnel

iv. American Medical Association-Mini-Z Burnout Inventory (Screening/Survey Instrument )

https://www.stepsforward.org/Static/images/modules/15/downloadable Mini\%20Z\%20burnout\%20survey.docx

v. Professional Quality of Life Scale (PROQOL) 33-item scale measuring compassion satisfaction, burnout and secondary traumatic stress http://www.proqol.org/uploads/ProQOL 5 English Self-Score 3 2012.pdf

vi. Depression screen:

http://www.phqscreeners.com/sites/g/files/g10016261/f/201412/PHQ 9 English.pdf

vii. Mental Health America: http://www.mentalhealthamerica.net/mental-

B iii. Substance Abuse, Depression and Addressing Sequelae of Burnout ${ }^{14}$

2. https://sites.duke.edu/thelifecurriculum/files/2014/05/Macy-

5. Screening and Assessment Tools in place, cardiothoracic training programs will have to determine how the content should be delivered, ie, grand rounds, small-group discussions, or online educational modules like those of the AMA. ${ }^{19}$ Cardiothoracic training programs will have to determine how frequently the content is delivered and updated. The rigor of a program's wellness education could potentially be tested in in-service examinations, board examinations, or ACGME site visits. We recommend that programs designate a faculty member to oversee the wellness curriculum and the delivery of the content.

Another ACGME core requirement for training programs is access to tools for screening and assessment of wellness, burnout, depression, or professional quality of life. We list some online screening tools in the template policy (Figure 3, A-D). At this time, there are no guidelines on who should administer the screening, who should be screened (residents only vs residents and faculty), optimal interval of screening, and what to do with the results. At the May 2019 Thoracic Surgery Directors Association meeting, program directors discussed the risks of selfassessment. Should the results be individual and confidential? If so, would a trainee who has burnout then be responsible for self-navigating to seek help? Alternatively, programs could ask trainees to share self-screening results. However, this practice cannot conflict with each trainees' right to health privacy. We advise that a wellness policy specifically determine timing of assessment, which assessment tool will be used, who is to administer the assessment, how results of these screening instruments are used, and how each individual's wellness is part of or distinctly separate from trainees' overall evaluation.

Wellness programs should not only provide access to health care but also foster a culture of attention to health maintenance. Programs must ensure adequate sleep facilities, safe work environment, access to water and healthy food, and safe transportation options for trainees who may be too fatigued to safely return home. Residents must have the opportunity to attend medical, mental health, and dental care appointments, and programs must adjust workload and schedules accordingly. Hopefully, all trainees will take advantage of scheduled time for health maintenance without feeling guilty about leaving work. 

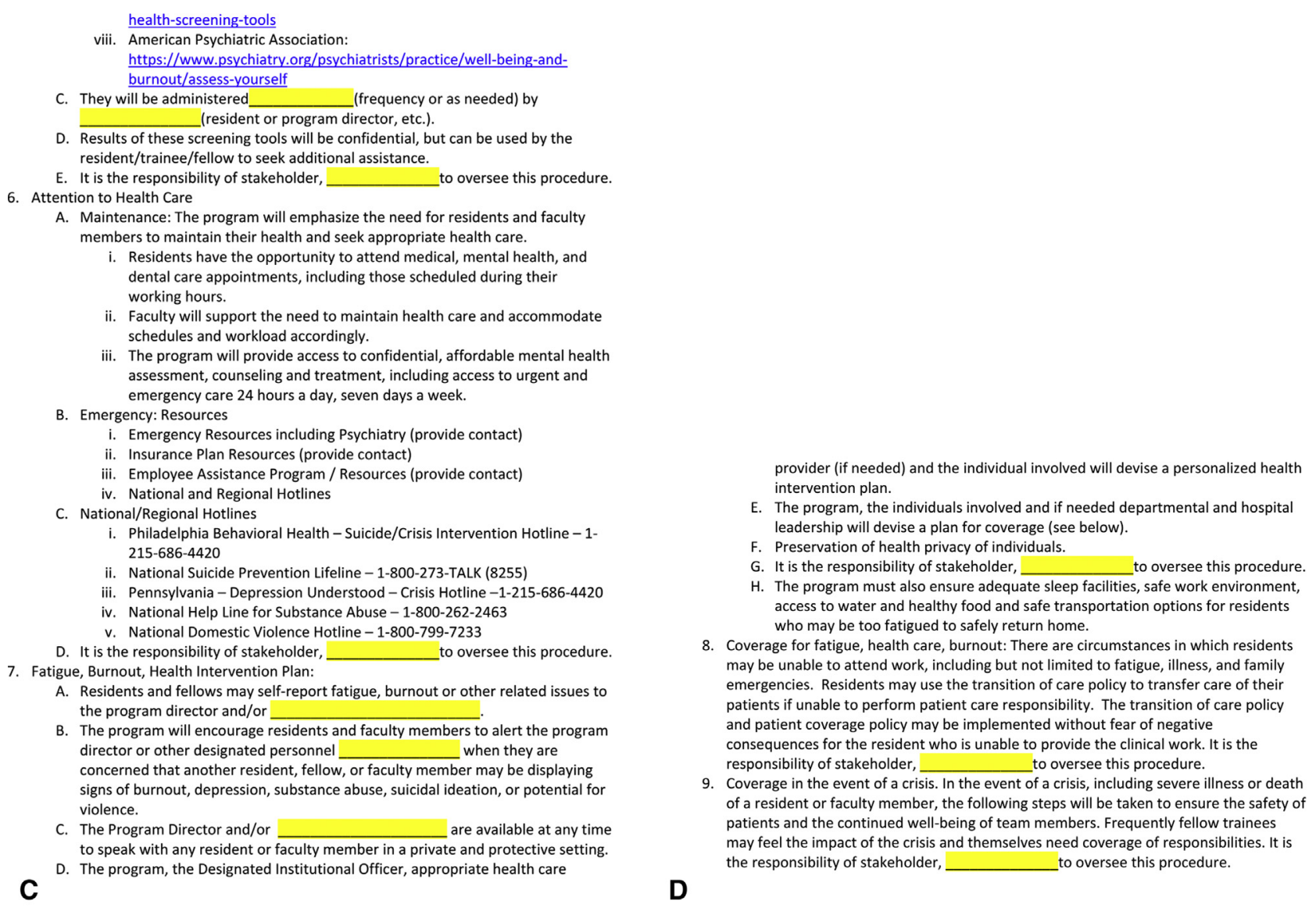

FIGURE 3. Continued

Wellness programs should have specific plans to intervene with fatigue, burnout, substance abuse, potential for harm to self or others, or other health needs including pregnancy, maternity/paternity leave, and family medical leave. Faculty and trainees must report fatigue, burnout, or other health issues to the program director and/or an alternative person responsible for wellness. In turn, program directors must develop an expeditious plan to address the needs of the individual. For mental health concerns, program directors must provide contact information for 24-hour-a-day resources (mental health assessment, counseling, treatment). This care should be confidential and affordable. Academic institutions and health systems may have established resources available to faculty and trainees. Program directors should also include a plan for coverage of patient care if a resident requires an intermittent, short-term, or long-term time away from training. This plan should relate to each program's existing Transition of Care Policy. At times, the individual experiencing fatigue or burnout may not be able to arrange a safe transition of care and responsibilities; in this circumstance program leadership should organize coverage. It is advisable to have a crisis plan in the event of a severe illness, prolonged absence, or death. Such events may require that co-trainees and possibly faculty take time away from clinical responsibilities to cope with stress and grief. Having a policy of coverage, which may include increased responsibility on the part of faculty, may facilitate arrangements for particularly trying times. As with other wellness procedures, a designated person should be responsible for updating wellness resources and procedures. ${ }^{25}$

This checklist and sample wellness policy have limitations. They have been developed in response to the ACGME Core Program Requirements using resources developed for physicians. Further study into specific wellness issues for cardiothoracic trainees is needed. It is also unknown how effective these generalized wellness procedures are in the setting of cardiothoracic training. It is unknown how cardiothoracic surgeons will engage in wellness, even with mandates from the ACGME or program leadership. Furthermore, it is difficult for programs to mobilize resources to develop and maintain a wellness policy. In particular, education of faculty and residents about wellness, fatigue, and burnout is a daunting responsibility for program directors. 


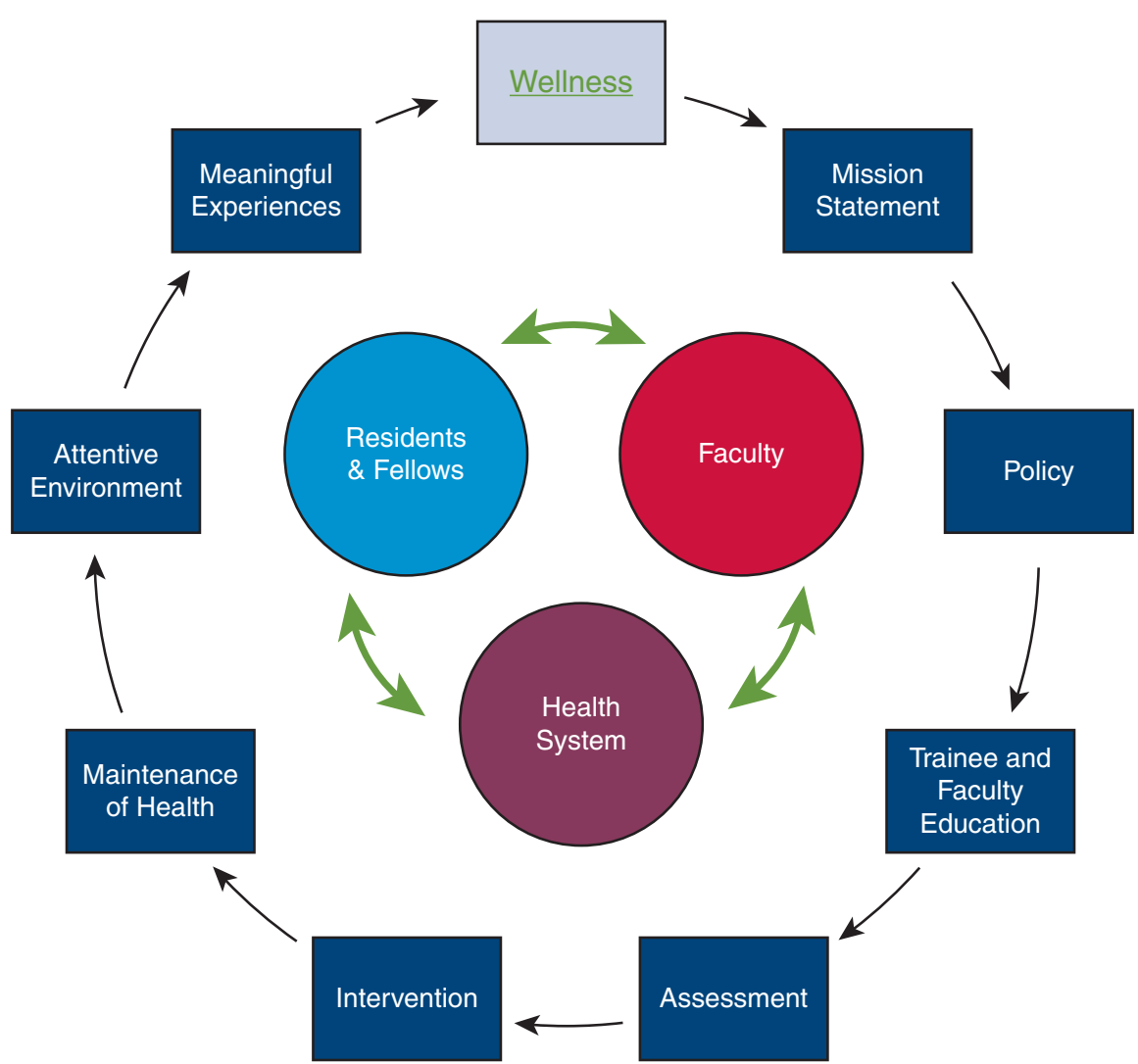

FIGURE 4. Implementation of wellness. The outer circle represents the key components in the process of implementing wellness. The inner circle comprises all the key stakeholders involved with the implementation of wellness and their interdisciplinary relationship (green arrows).

We represent 3 different ACGME thoracic surgery training programs with different resources, needs, and stages of development in regard to wellness. However, shared barriers that we encountered include the following: (1) Prioritizing what can be done to support a culture of wellness. (2) Finding resources and time to implement measures. (3) Finding a meaningful way to improve wellness among faculty and trainees, without making it yet another responsibility they have to fulfill. To address program directors' new responsibility of implementing wellness into cardiothoracic surgery programs, we have delineated the requirements, developed a wellness program checklist (Figure 2) and developed a sample wellness policy template (Figure $3, A-D$ ). Our hope is that we can facilitate organization of wellness and provide a shared framework for building a culture of wellness. These tools will not only assist in compliance with ACGME Well-Being Requirements, but also ensure a comprehensive and program-specific wellness program that applies to each program's trainees and faculty (Figure 4). As programs adopt wellness, hopefully we can develop a means of sharing experiences and resources. Potential next steps could include a shared cardiothoracic surgery wellness curriculum, website with a listing of resources and assessment tools, and educational workshops either online or at national meetings. This would not only minimize the burden of program directors but also ensure consistency of content. Hopefully leadership at each institution and our national cardiac and thoracic community will support the added responsibilities of promoting wellness.

\section{CONCLUSIONS}

Physician well-being optimizes safe patient care and education. We developed a checklist and a sample wellness policy for cardiac and thoracic training programs that incorporates the ACGME Well-Being Requirements. These tools will give programs a structure to implement wellness while incorporating a mission, personnel, and procedures specific to each program.

\section{Conflict of Interest Statement}

The authors reported no conflicts of interest.

The Journal policy requires editors and reviewers to disclose conflicts of interest and to decline handling or reviewing manuscripts for which they may have a conflict of interest. The editors and reviewers of this article have no conflicts of interest. 


\section{References}

1. Schwenk TL, Davis L, Wimsatt LA. Depression, stigma, and suicidal ideation in medical students. JAMA. 2010;304:1181-90.

2. Dyrbye LN, Thomas MR, Massie FS, Power DV, Eacker A, Harper W, et al. Burnout and suicidal ideation among U.S. medical students. Ann Intern Med. 2008; 149:334.

3. Prins JT, Gazendam-Donofrio SM, Tubben BJ, van der Heijden FM, van de Wiel HB, Hoekstra-Weebers JE. Burnout in medical residents: a review. Med Educ. 2007;41:788-800.

4. Mata DA, Ramos MA, Bansal N, Khan R, Guille C, Di Angelantonio E, et al. Prevalence of depression and depressive symptoms among resident physicians: a systematic review and meta-analysis. JAMA. 2015;314:2373-83.

5. Balch CM, Shanafelt TD, Sloan JA, Satele DV, Freischlag JA. Distress and career satisfaction among 14 surgical specialties, comparing academic and private practice settings. Ann Surg. 2011;254:558-68.

6. Shanafelt TD, Balch CM, Bechamps GJ, Russell T, Dyrbye L, Satele D, et al. Burnout and career satisfaction among American surgeons. Ann Surg. 2009; 127:107-15.

7. Panagioti M, Geraghty K, Johnson J, Zhou A, Panagopoulou E, Chew-Graham C, et al. Association between physician burnout and patient safety, professionalism, and patient satisfaction: a systematic review and meta-analysis. JAMA Intern Med. 2018;178:1317-30.

8. West CP, Huschka MM, Novotny PJ, Sloan JA, Kolars JC, Habermann TM, et al. Association of perceived medical errors with resident distress and empathy. JAMA. 2006;296:1071.

9. Han S, Shanafelt TD, Sinsky CA, Awad KM, Dyrbye LN, Fiscus LC, et al. Estimating the attributable cost of physician burnout in the United States. Ann Intern Med. 2019;170:784-90.

10. Accreditation Council for Graduate Medical Education. Improving Physician Well-Being, Restoring Meaning in Medicine. Available at: https://www.acgme. org/What-We-Do/Initiatives/Physician-Well-Being. Accessed November 11, 2019.

11. American Medical Association. Preventing burnout in medical residents and fellows: 6 keys for wellness. Available at: https://www.ama-assn.org/residentsstudents/resident-student-health/preventing-burnout-medical-residents-andfellows-6-keys. Accessed November 11, 2019.

12. Fletcher KE, Reed DA, Arora VM. Doing the dirty work: measuring and optimizing resident workload. J Gen Intern Med. 2011;26:8-9.
13. Thanarajasingam U, McDonald FS, Halvorsen AJ, Naessens JM, Cabanela RL Johnson MG, et al. Service census caps and unit-based admissions: resident workload, conference attendance, duty hour compliance, and patient safety. Mayo Clin Proc. 2012;87:320-7.

14. Ratcliffe TA, Crabtree MA, Palmer RF, Pugh JA, Lanham HJ, Leykum LK. Service and education: the association between workload, patient complexity, and teaching on internal medicine inpatient services. J Gen Intern Med. 2018; 33:449-54.

15. Kaushal A, Katznelson L, Harrington RA. Beyond duty hours: leveraging large-scale paging data to monitor resident workload. NPJ Digit Med. 2019; 2:87.

16. Blum AB, Shea S, Czeisler CA, Landrigan CP, Leape L. Implementing the 2009 Institute of Medicine recommendations on resident physician work hours, supervision, and safety. Nat Sci Sleep. 2011;3:47-85.

17. American College of Surgeons. Surgeon well-being. Available at: https://www. facs.org/member-services/surgeon-wellbeing. Accessed November 11, 2019

18. Raj KS. Well-being in residency: a systematic review. J Grad Med Educ. 2016;8: 674-84.

19. American Medical Association Ed Hub. Burnout and well-being. Available at: https://edhub.ama-assn.org/steps-forward/pages/professional-well-being. Accessed November 20, 2019

20. Duke School of Medicine. L.I.F.E. teacher's guide. Available at: https://sites.duke. edu/thelifecurriculum/files/2014/05/Macy-teachersguide1.pdf. Accessed November 20, 2019.

21. Mayo Clinic. Make the Difference: Preventing Medical Trainee Suicide. Available at: https://www.youtube.com/watch?v=I9GRxF9qEBA. Accessed November 20, 2019

22. Hill AB. Breaking the stigma-a physician's perspective on self-care and recovery. N Engl J Med. 2017;376:1103-5.

23. Kalmoe MC, Chapman MB, Gold JA, Giedinghagen AM. Physician suicide: a call to action. Mo Med. 2019;116:211-6.

24. Thoracic Surgery Directors Association. Fighting fatigue: a guide for cardiothoracic surgery residents. Available at: https://www.tsranet.org/wp-content/ uploads/2013/01/Fighting_Fatigue.pdf. Accessed November 20, 2019.

25. National Academy of Medicine. Action Collaborative on Clinician Well-Being and Resilience. Available at: https://nam.edu/initiatives/clinician-resilience-and-wellbeing/commitment-statements-clinician-well-being/. Accessed November 22, 2019 . 